\title{
The World Health Organization standards for tuberculosis care and management
}

\author{
Christopher Gilpin ${ }^{1}$, Alexei Korobitsyn ${ }^{1}$, Giovanni Battista Migliori (i) ${ }^{2}$, \\ Mario C. Raviglione ${ }^{3}$ and Karin Weyer ${ }^{1}$
}

Affiliations: ${ }^{1}$ Global Tuberculosis Programme, World Health Organization, Geneva, Switzerland. ${ }^{2}$ World Health Organization Collaborating Centre for Tuberculosis and Lung Diseases, Maugeri Care and Research Institute, IRCCS, Tradate, Italy. ${ }^{3}$ University of Milan, Dept of Global Health, Milan, Italy.

Correspondence: Giovanni Battista Migliori, WHO Collaborating Centre for TB and Lung Diseases, Fondazione S. Maugeri, Care and Research Institute, via Roncaccio 16, 21049 Tradate, Italy.

E-mail: giovannibattista.migliori囚icsmaugeri.it

\section{@ERSpublications}

The WHO TB Compendium facilitates the delivery of high-quality care to all persons affected by TB http://ow.ly/VzsM30iO8sO

Cite this article as: Gilpin C, Korobitsyn A, Migliori GB, et al. The World Health Organization standards for tuberculosis care and management. Eur Respir J 2018; 51: 1800098 [https://doi.org/10.1183/ 13993003.00098-2018].

Tuberculosis (TB) is a global public health priority, with 10.4 million people developing the disease in 2016 (10\% among HIV co-infected individuals), 1.67 million deaths and 490000 multidrug-resistant (MDR) plus an additional 110000 rifampicin-resistant cases [1].

In 2014, the World Health Organization (WHO) launched the End TB Strategy [1] within the framework of the United Nations Sustainable Development Goals (SDGs). The new strategy was designed to be consistent with the preceding WHO-recommended strategies for TB care and control [2]: the DOTS strategy (launched in 1994, creating the basis for effective TB care and management activities by standardising the requirements to address the epidemic) and the Stop TB Strategy (launched in 2006, aimed at tackling the emerging priorities, such as MDR-TB and TB/HIV co-infection).

In order to end the TB epidemic (which is one of the SDG targets) several interventions need to be implemented. They include the core anti-TB interventions (diagnosis, treatment and prevention), which are described under Pillar I of the Strategy; and several socioeconomic and public health interventions, which are often under the responsibility of ministries other than the ministry of health (e.g. economy, finance, interior or justice) and are presented under Pillar II. Intensified research activities and innovation are covered by Pillar III.

The multidimensional interventions of the End TB Strategy and their potential impact on the TB epidemic are summarised in figure 1, and apply to both high and low TB and HIV burden settings. In countries at low TB incidence ( $<10$ cases per 100000 population), optimal application of the principles of the strategy should allow approach of the pre-elimination threshold $(<10 \mathrm{~TB}$ cases per million population) and hopefully also the TB elimination one ( $<1$ TB case per million) by 2035 [4-7].

In November 2017, WHO launched a core document: the "Compendium of WHO guidelines and associated standards: ensuring optimum delivery of the cascade of care for patients with tuberculosis", hereafter referred to as the Compendium. It is structured into WHO-recommended TB standards and 


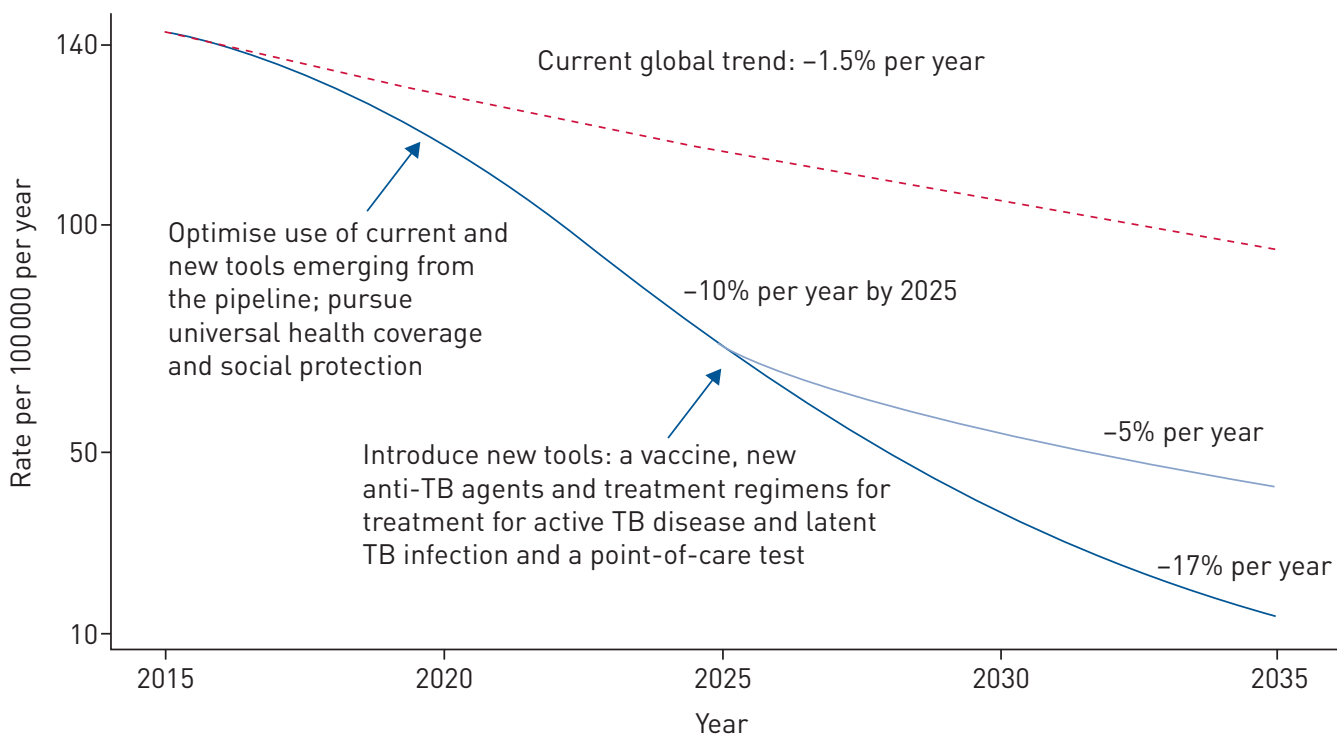

FIGURE 1 Projected global trajectory of tuberculosis (TB) incidence rate 2015-2035 required to reach 2035 targets of the End TB Strategy. Reproduced and modified from [3] with permission.

consolidates all current WHO TB policy recommendations into a single resource. The document thus provides a framework for reaching the ambitious targets in the End TB Strategy [3].

The aim of this editorial is to introduce this comprehensive resource to a wide audience. The Compendium is relevant for all healthcare providers (clinically oriented as well as public health-oriented readers) in both the private and public sectors.

The document describes the principles on which the End TB Strategy is based (such as ethical considerations, community engagement, public-private mix and engaging all care providers) and provides insight to the formal WHO policy development process. Furthermore, the Compendium covers the activities of Pillar I of the End TB Strategy, focusing on integrated, patient-centred diagnosis, treatment and prevention of TB in adults and children, within close collaboration involving civil society, the social sector, local communities and other stakeholders [3].

The Compendium has been developed as a clear and concise instrument to facilitate the understanding and planning of delivery of high-quality care for everybody affected by TB. It follows the pathway of persons with signs or symptoms of $\mathrm{TB}$ in seeking diagnosis, treatment and care, and includes key algorithms and cross-cutting elements that are essential to a patient-centred approach in the cascade of TB care.

Beyond accelerated implementation of existing tools, an effective TB response must embrace innovation through the rapid uptake of new interventions such as rapid diagnostics, new medicines and digital platforms to modernise care provision. Working with communities, civil society and all partners, governments need to assume full responsibility for ensuring person-centred, modern, high-quality TB services and securing comprehensive care along with essential support for each person with $\mathrm{TB}$, which also calls for collaboration within and beyond the health sector. The Compendium is consistent with the principles of International Standards of TB Care [8] and with the European Standards for TB Care [9] in stressing the need to engage all care providers regardless of whether care is sought from public, voluntary, private or corporate care providers.

The Compendium will be updated regularly, including in digital format, to allow incorporation of new evidence emerging from the rapidly evolving TB diagnostic and treatment landscape.

The Compendium includes 33 standards (tables 1 and 2). Table 1 presents the WHO standards for early TB detection, reminding clinicians and other healthcare providers that prompt clinical evaluation for $\mathrm{TB}$ is essential (standards 1-4). Standards 5-10 are focused on the importance of quality-assured diagnostic tests for the detection of drug-susceptible and drug-resistant TB. Standards 11 and 18 provide guidance for the diagnosis and treatment of latent TB infection. Standards 12-17 relate to the treatment of both drug-susceptible and drug-resistant TB cases. 
TABLE 1 Summary of the World Health Organization (WHO) standards for early detection, diagnosis and treatment of tuberculosis (TB) (susceptible and drug-resistant) and latent tuberculosis infection (LTBI)

\section{Standards for early TB detection}

1) For persons with signs or symptoms consistent with $T B$, performing prompt clinical evaluation is essential to ensure early and rapid diagnosis $[10,11]$.

2) All persons who have been in close contact with patients who have pulmonary TB should be evaluated. The highest priority contacts for evaluation are those [3]:

with signs or symptoms suggestive of TB;

aged $<5$ years;

with known or suspected immunocompromising conditions, particularly HIV infection;

who have been in contact with patients with MDR-TB or XDR-TB.

3) All persons living with HIV and workers who are exposed to silica should always be screened for active TB in all settings. Other high-risk groups should be prioritised for screening based on the local TB epidemiology, health system capacity, resource availability and feasibility of reaching the risk groups [3, 12].

4) CXR is an important tool for triaging and screening for pulmonary TB, and it is also useful to aid diagnosis when pulmonary TB cannot be confirmed bacteriologically. CXR can be used to select individuals for referral for bacteriological confirmation and the role of radiology remains important when bacteriological tests cannot provide a clear answer $[3,13,14]$.

\section{Standards for TB diagnosis}

5) To safely and efficiently diagnose TB and drug-resistant TB requires a functional network of quality-assured laboratories with appropriate biosafety measures in place for performing different technical procedures. As such, TB programmes require a tiered network of integrated laboratories in which different levels use complementary tools to diagnose TB and HIV, and have mechanisms for referring specimens between the different levels of the network $[3,15]$.

6) All patients with signs and symptoms of pulmonary TB who are capable of producing sputum should have as their initial diagnostic test at least one sputum specimen submitted for Xpert MTB/RIF Ultra assay. This includes children who are able to provide a sputum sample and patients with EPTB. A second Xpert MTB/RIF Ultra assay may be performed for all patients who initially test negative by Xpert MTB/ RIF Ultra but whose signs and symptoms of TB persist $[3,16]$.

7) The Xpert MTB/RIF Ultra assay should be used in preference to conventional microscopy and culture as the initial diagnostic test for cerebrospinal fluid specimens from patients being evaluated for TB meningitis. The Xpert MTB/RIF Ultra assay is recommended as a replacement test for usual practice (including conventional microscopy, culture or histopathology) for testing specific nonrespiratory specimens (lymph nodes and other tissues) from patients suspected of having EPTB [3].

8) For persons living with HIV, the Xpert MTB/RIF Ultra assay should be used as an initial diagnostic test. LF-LAM can be used to assist in the diagnostic process for HIV-positive patients who are seriously ill [3, 17-19].

9) DST using WHO-recommended rapid tests should be performed for all TB patients prior to starting therapy, including new patients and patients who require retreatment. If rifampicin resistance is detected, rapid molecular tests for resistance to isoniazid, fluoroquinolones and second-line injectable agents should be performed promptly to inform the treatment of MDR-TB and XDR-TB [3, 20-22].

10) Culture-based DST for selected second-line anti-TB agents should be performed for patients enrolled in individualised (longer) MDR-TB treatment [3].

\section{Standard for diagnosing LTBI}

11) Either TST or IGRA can be used to test for LTBI. TST is not required before initiating IPT in persons living with HIV [23].

\section{Standards for treating drug-susceptible TB}

12) While awaiting DST results, patients with drug-susceptible TB and TB patients who have not been treated previously with anti-TB agents and do not have other risk factors for drug resistance should receive a WHO-recommended first-line treatment regimen using quality assured anti-TB agents. The initial phase should consist of 2 months of isoniazid, rifampicin, pyrazinamide and ethambutol. The continuation phase should consist of 4 months of isoniazid and rifampicin. Daily dosing should be used throughout treatment. The doses of anti-TB agents should conform to WHO's recommendations. FDC anti-TB agents may provide a more convenient form of administration [3, 24-26].

13) In patients who require retreatment for TB, the category II regimen should no longer be prescribed and DST should be conducted to inform the choice of treatment regimen [3].

14) In patients with tuberculous meningitis or tuberculous pericarditis, adjuvant corticosteroid therapy should be used in addition to an appropriate TB treatment regimen [3].

\section{Standards for treating drug-resistant TB}

15) In patients with rifampicin-susceptible, isoniazid-resistant TB, 6 months of combination treatment with rifampicin, ethambutol, pyrazinamide and levofloxacin, with or without isoniazid, is recommended $[3,25,26]$.

16) Patients with MDR/RR-TB require second-line treatment regimens. MDR/RR-TB patients may be treated using a 9-11-month MDR-TB treatment regimen (the shorter regimen) unless they have resistance to second-line anti-TB agents or meet other exclusion criteria. In these cases, a longer (individualised) regimen with at least five effective anti-TB agents in the intensive phase and four agents in the continuation phase is recommended for $\geqslant 20$ months. Partial resection surgery has a role in treating MDR-TB $[3,25]$.

17) A system to actively monitor and manage harms caused by anti-TB agents is required whenever drug-resistant TB patients are treated with novel or repurposed medicines and MDR-TB regimens $[3,27]$.

\section{Standard for treating LTB}

18) Persons living with HIV and children younger than 5 years who are household or close contacts of persons with TB and who, after an appropriate clinical evaluation, are found not to have active TB but to have LTBI should be treated [3, 23].

MDR: multidrug-resistant; XDR: extensively drug-resistant; CXR: chest radiography; EPTB: extrapulmonary tuberculosis; LF-LAM: lateral-flow urine lipoarabinomannan assay; DST: drug susceptibility testing; TST: tuberculin skin test; IGRA: interferon- $\gamma$ release assay; IPT: isoniazid preventative therapy; FDC: fixed-dose combination; RR: rifampicin-resistant. 
TABLE 2 Summary of the World Health Organization standards for HIV infection and other comorbid conditions, tuberculosis (TB) in children, monitoring and evaluation activities, and supportive approaches to the management of TB patients

\section{Standards for HIV infection and other comorbid conditions}

19) HIV testing should be routinely offered to all patients with presumptive TB and those who have been diagnosed with TB $[3,28]$.

20) Persons living with HIV should be screened for TB by using a clinical algorithm [3].

21) ART and routine CPT should be initiated among all TB patients living with HIV, regardless of their CD4 cell count [3].

22) A thorough assessment should be conducted to evaluate comorbid conditions and other factors that could affect the response to or outcome of TB treatment. Particular attention should be given to diseases or conditions known to affect treatment outcomes, such as diabetes mellitus, drug and alcohol abuse, undernutrition and tobacco smoking [3].

\section{Standards for managing TB in children}

23) The diagnosis of TB in children relies on the thorough assessment of all evidence derived from a careful history (including history of TB contacts and symptoms consistent with TB), clinical examination (including growth assessment), a TST, CXR (if available), bacteriological confirmation whenever possible, investigations for suspected pulmonary TB and suspected EPTB, and HIV testing. Whenever possible, the Xpert MTB/RIF Ultra assay should be used as the initial diagnostic test in children suspected of having any form of TB [29].

24) The principles of treating TB in children are the same as for treating TB in adults: first-line treatment of drug-sensitive TB consists of a 2-month intensive phase with isoniazid, rifampicin, pyrazinamide and, depending on the setting and type of disease, ethambutol, followed by a continuation phase with isoniazid and rifampicin for $\geqslant 4$ months; however, the dose of first-line anti-TB agents differs from that administered in adults [3].

25) In settings where TB is highly endemic or where there is a high risk of exposure to TB, a single dose of BCG vaccine should be given to all infants; however, HIV-positive children should not be given BCG vaccine. After considering local factors, BCG vaccine should be given to all infants except those who are HIV-positive for whom BCG is contraindicated [3].

26) All children younger than 5 years and HIV-positive children of any age should be included in contact screening and management efforts, with the aim of identifying undiagnosed TB disease and providing preventive therapy for contacts without TB disease that are susceptible to developing disease following exposure to a contact with active TB disease [3].

\section{Standard for monitoring and evaluation}

27) All providers must report both new and re-treatment TB cases and their treatment outcomes to national public health authorities in conformance with applicable legal requirements and policies; TB mortality should be monitored by using standard cause-of-death data from vital registration systems $[3,30]$.

\section{Standards for supportive approaches to TB patient management}

28) Digital technologies can be adapted to increase the effectiveness or efficiency of different components of TB programmes [3, 24].

29) Promptly identify persons with TB symptoms (triage); provide an adequately ventilated waiting area for them; educate them about cough etiquette and respiratory hygiene; ensure they are prioritised for TB testing; and separate infectious patients [3].

30) A patient-centred approach to treatment should be developed to promote adherence, improve quality of life and relieve suffering. This approach should be based on the patient's needs and on mutual respect between the patient and the provider [3, 24].

31) Prior to starting TB treatment, each patient's need for support should be assessed, and interventions to encourage adherence to treatment be offered to improve outcomes $[3,24]$.

32) Before starting TB treatment, all patients should be assessed to determine the risk of treatment interruption and appropriate options for treatment administration should be offered to each patient. Community- or home-based DOT is recommended over health facility-based DOT or unsupervised treatment; and DOT administered by trained lay providers or healthcare workers is recommended over DOT administered by family members. Video-observed treatment may replace DOT when the technology is available, and can be organised and operated by healthcare providers and patients $[3,24]$.

33) All forms of suffering associated with TB should be addressed by ensuring that patients have proper access to care, and to the management of adverse reactions to treatment, management of psychological distress, means to prevent and mitigate stigma and discrimination; and by providing access to social protection mechanisms to reduce indirect costs [3].

ART: antiretroviral therapy; CPT: co-trimoxazole preventive therapy; TST: tuberculin skin test; CXR: chest radiography; EPTB: extrapulmonary tuberculosis; BCG: bacille Calmette-Guérin; DOT: directly observed therapy.

Table 2 presents the summary of the WHO standards for TB comorbid conditions (standards 19-22), TB in children (standards 23-26), monitoring and evaluation activities (standard 27), and supportive approaches to the care of TB patients (standards 28-33).

Given that the Compendium represents a consolidated document summarising all current WHO policies and supportive approaches for TB diagnosis treatment and care delivery, it will be useful to inform national TB programmes when drafting local adaptations of WHO specific guidelines. It should also serve as a resource for private sector clinicians to support their care delivery for all patients affected by TB.

Acknowledgements: The contributors to the Compendium are Annabel Baddeley, Annemieke Brands, Monica Dias, Dennis Falzon, Haileyesus Getahun, Christopher Gilpin, Philippe Glaziou, Malgosia Grzemska, Yohhei Hamada, Ernesto Jaramillo, Alexei Korobitsyn, Knut Lönnroth, Fuad Mirzayev, Linh Nguyen, Kefas Samson, Lana Syed, Mario Raviglione, Hazim Timimi, Mukund Uplekar, Wayne van Gemert, Diana Weil, Karin Weyer and Matteo Zignol (all Global Tuberculosis Programme, WHO, Geneva, Switzerland).

Conflict of interest: None declared. 


\section{References}

1 World Health Organization. Global Tuberculosis Report 2017. Document WHO/HTM/TB/2017.23. Geneva, World Health Organization, 2017. Available from: http://apps.who.int/iris/bitstream/10665/259366/1/ 9789241565516-eng.pdf?ua=1. Date last accessed: January 15, 2018.

2 Sotgiu G, Spanevello A, Migliori GB. History of tuberculosis and drug resistance. N Engl J Med 2013; 368: 88-89.

3 World Health Organization. Compendium of WHO guidelines and associated standards: ensuring optimum delivery of the cascade of care for patients with tuberculosis. Document WHO/HTM/TB/2017.13. Geneva, World Health Organization, 2017. Available from: http://apps.who.int/iris/bitstream/10665/259180/1/9789241512572-eng. pdf. Date last accessed: January 15, 2018.

4 Lönnroth K, Migliori GB, Abubakar I, et al. Towards tuberculosis elimination: an action framework for low-incidence countries. Eur Respir J 2015; 45: 928-952.

5 Voniatis C, Migliori GB, Voniatis M, et al. Tuberculosis elimination: dream or reality? The case of Cyprus. Eur Respir J 2014; 44: 543-546.

6 Al Yaquobi F, Al-Abri S, Al-Abri B, et al. TB elimination, dream or reality? The case of Oman. Eur Respir J 2018; 51: 1702027.

7 Blasi F, Matteeelli A, Sotgiu G, et al. Moving towards tuberculosis elimination: a call for action from Italy. Eur Respir J 2017; 49: 1602242.

8 TB CARE I. International Standards for Tuberculosis Care. 3rd Edn. The Hague, TB CARE, 2014. Available from: www.who.int/tb/publications/standards-tb-care-2014/en/. Date last accessed: January 15, 2018.

9 Migliori GB, Zellweger JP, Abubakar I, et al. European union standards for tuberculosis care. Eur Respir J 2012; 39: $807-819$.

10 World Health Organization. Early detection of tuberculosis: an overview of approaches, guidelines and tools. Document WHO/HTM/STB/PSI/2011.21. Geneva, World Health Organization, 2011. Available from: http://apps.who. int/iris/bitstream/10665/70824/1/WHO_HTM_STB_PSI_2011.21_eng.pdf. Date last accessed: January 15, 2018.

11 World Health Organization. Recommendations for investigating contacts of persons with infectious tuberculosis in low- and middle-income countries. Document WHO/HTM/TB/2012.9. Geneva, World Health Organization, 2012. Available from: http://apps.who.int/iris/bitstream/10665/77741/1/9789241504492_eng.pdf?ua=1. Date last accessed: January 15, 2018.

12 World Health Organization. Systematic screening for active tuberculosis. Principles and recommendations. Document WHO/HTM/TB/2013.04. Geneva, World Health Organization, 2013. Available from: http://apps.who. int/iris/bitstream/10665/84971/1/9789241548601_eng.pdf?ua=1\&ua=1. Date last accessed: January 15, 2018.

13 World Health Organization. Chest radiography in tuberculosis detection. Summary of current WHO recommendations and guidance on programmatic approaches. Document WHO/HTM/TB/2016.20. Geneva, World Health Organization, 2016. Available from: http://apps.who.int/iris/bitstream/10665/252424/1/ 9789241511506-eng.pdf?ua=1. Date last accessed: January 15, 2018.

14 Dara M, Sotgiu G, Zaleskis R, et al. Untreatable tuberculosis: is surgery the answer? Eur Respir J 2015; 45: $577-582$.

15 World Health Organization. Implementing tuberculosis diagnostics: policy framework. Document WHO/HTM/ TB/2015.11. Geneva, World Health Organization, 2015. Available from: http://apps.who.int/iris/bitstream/10665/ 162712/1/9789241508612_eng.pdf?ua=1\&ua=1. Date last accessed: January 15, 2018.

16 World Health Organization. Xpert MTB/RIF implementation manual. Technical and operational 'how-to': practical considerations. Document WHO/HTM/TB/2014.1. Geneva, World Health Organization, 2014. Available from: http://apps.who.int/iris/bitstream/10665/112469/1/9789241506700_eng.pdf?ua=1. Date last accessed: January $15,2018$.

17 World Health Organization. WHO meeting report of a technical expert consultation: non-inferiority analysis of Xpert MTB/RIF Ultra compared to Xpert MTB/RIF. Document WHO/HTM/TB/2017.04. Geneva, World Health Organization, 2017. Available from: http://apps.who.int/iris/bitstream/10665/254792/1/WHO-HTM-TB-2017. 04-eng.pdf. Date last accessed: January 15, 2018.

18 World Health Organization. The use of loop-mediated isothermal amplification (TB-LAMP) for the diagnosis of pulmonary tuberculosis: policy guidance. Document WHO/HTM/TB/2016.11. Geneva, World Health Organization, 2016. Available from: http://apps.who.int/iris/bitstream/10665/249154/1/9789241511186-eng.pdf? ua $=1$. Date last accessed: January 15, 2018.

19 World Health Organization. The use of lateral flow urine lipoarabinomannan assay (LF-LAM) for the diagnosis and screening of active tuberculosis in people living with HIV: policy guidance. Document WHO/HTM/TB/ 2015.25. Geneva, World Health Organization, 2015. Available from: http://apps.who.int/iris/bitstream/10665/ 193633/1/9789241509633_eng.pdf?ua=1\&ua=1. Date last accessed: January 15, 2018.

20 World Health Organization. Considerations for adoption and use of multi-disease testing devices in integrated laboratory networks. Document WHO/HTM/TB/2017.06. Geneva, World Health Organization, 2017. Available from: http://apps.who.int/iris/bitstream/10665/255693/1/WHO-HTM-TB-2017.06-eng.pdf. Date last accessed: January 15, 2018.

21 World Health Organization. The use of molecular line probe assays for the detection of resistance to isoniazid and rifampicin: policy update. Geneva, 2016 update. Document WHO/HTM/TB/2016.12. Geneva, World Health Organization, 2016. Available from: http://apps.who.int/iris/bitstream/10665/250586/1/9789241511261-eng.pdf? ua $=1$. Date last accessed: January 15, 2018.

22 World Health Organization. The use of molecular line probe assays for the detection of resistance to second-line anti-tuberculosis drugs: policy guidance. Document WHO/HTM/TB/2016.07. Geneva, World Health Organization, 2016. Available from: http://apps.who.int/iris/bitstream/10665/246131/1/9789241510561-eng.pdf? ua $=1$. Date last accessed: January 15, 2018.

23 Getahun H, Matteelli A, Abubakar I, et al. Management of latent Mycobacterium tuberculosis infection: WHO guidelines for low tuberculosis burden countries. Eur Respir J 2015; 46: 1563-1576.

24 World Health Organization. Guidelines for treatment of drug-susceptible tuberculosis and patient care: 2017 update. Document WHO/HTM/TB/2017.05. Geneva, World Health Organization, 2017. Available from: http:// apps.who.int/iris/bitstream/10665/255052/1/9789241550000-eng.pdf?ua=1. Date last accessed: January 15, 2018. 
25 Falzon D, Schünemann HJ, Harausz E, et al. World Health Organization treatment guidelines for drug-resistant tuberculosis, 2016 update. Eur Respir J 2017; 49: 1602308.

26 World Health Organization. Companion handbook to the WHO guidelines for the programmatic management of drug-resistant tuberculosis. Document WHO/HTM/TB/2014.11. Geneva, World Health Organization, 2014. Available from: http://apps.who.int/iris/bitstream/10665/130918/1/9789241548809_eng.pdf. Date last accessed: January 15, 2018.

27 World Health Organization. Active tuberculosis drug-safety monitoring and management (aDSM). Document WHO/HTM/TB/2015.28. Geneva, World Health Organization, 2015. Available from: http://apps.who.int/iris/ bitstream/10665/204465/1/WHO_HTM_TB_2015.28_eng.pdf?ua=1. Date last accessed: January 15, 2018.

28 World Health Organization. Integrating collaborative TB and HIV services within a comprehensive package of care for people who inject drugs: consolidated guidelines. Document WHO/HTM/TB/2016.02. Geneva, World Health Organization, 2016. Available from: http://apps.who.int/iris/bitstream/10665/204484/1/9789241510226_eng. pdf?ua=1. Date last accessed: January 15, 2018.

29 World Health Organization. Guidance for national tuberculosis programmes on the management of tuberculosis in children. 2nd Edn. Document WHO/HTM/TB/2014.03. Geneva, World Health Organization, 2014. Available from: http://apps.who.int/iris/bitstream/10665/112360/1/9789241548748_eng.pdf?ua=1. Date last accessed: January $15,2018$.

30 World Health Organization. Definitions and reporting framework for tuberculosis - 2013 revision. Updated December 2014. Document WHO/HTM/TB/2013.2. Geneva, World Health Organization, 2013. Available from: http://apps.who.int/iris/bitstream/10665/79199/1/9789241505345_eng.pdf. Date last accessed: January 15, 2018. 